\title{
Projeção da estrutura diamétrica em povoamentos de Eucalyptus grandis x urophylla submetidos a desbastes na Bahia
}

\author{
Projection of the diameter structure in Eucalyptus grandis x urophylla \\ stands submitted to thinnings in Bahia State

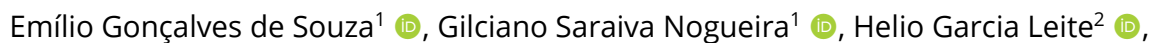 \\ Marcio Leles Romarco de Oliveira ${ }^{1}$ (1), Gabriela Paranhos Barbosa ${ }^{1}$ (), Efigênio Teixeira Lopes ${ }^{1}$ (1) \\ ${ }^{1}$ Universidade Federal dos Vales do Jequitinhonha e Mucuri - UFVJM, Diamantina, MG, Brasil \\ ${ }^{2}$ Universidade Federal de Viçosa - UFV, Viçosa, MG, Brasil
}

Como citar: Souza, E. G., Nogueira, G. S., Leite, H. G., Oliveira, M. L. R., Barbosa, G. P., \& Lopes, E. T. (2020).

Projeção da estrutura diamétrica em povoamentos de Eucalyptus grandis $x$ urophylla submetidos a desbastes na Bahia. Scientia Forestalis, 48(128), e3190. https://doi.org/10.18671/scifor.v48n128.07

\begin{abstract}
Resumo
Este trabalho teve como objetivo comparar dois modelos de distribuição de diâmetros para povoamentos de Eucalyptus grandis x urophylla submetidos a desbastes; e avaliar a influência de dois métodos de ajuste de funções densidade de probabilidade na qualidade das projeções de estruturas diamétricas. Os dados utilizados neste estudo foram provenientes de um experimento de clones de eucalipto, localizado na região Nordeste do estado da Bahia. As medições foram realizadas nas idades $27,40,50,58,61,76,87,101,112,122,137,147,158$ e 165 meses, com desbastes realizados aos 58 e 142 meses. Dois modelos de distribuição de probabilidade foram utilizados para prognosticar as estruturas diamétricas dos povoamentos, partindo-se de uma distribuição com base em dados observados nas idades 27 (antes do primeiro desbaste), 61 (após o primeiro desbaste) e 147 (após o segundo desbaste) meses. Foram obtidos três sistemas de acordo com o método de ajuste da função Weibull: Sistema 1, utilizando a aproximação linear; Sistemas 2 e 3, utilizando a máxima verossimilhança. A aderência das estruturas prognosticadas em relação às observadas foi verificada mediante o teste de Kolmogorov-Smirnov a 5\% de significância. O Sistema 2 foi o mais adequado para aplicações práticas com desbastes em eucalipto. O método da máxima verossimilhança deve ser preferencialmente utilizado quando comparado à aproximação linear, garantindo melhor qualidade nas prognoses da distribuição diamétrica.
\end{abstract}

Palavras-chave: Função Weibull; Distribuição de diâmetros; Método da máxima verossimilhança; Método da aproximação linear.

\begin{abstract}
This study aimed to compare two diameter distribution models in Eucalyptus grandis x Eucalyptus urophylla submitted to thinning; and evaluate the influence of two adjustment methods of probability density functions on the quality of the diameter structure projections. The data used in this study came from an experiment on thinning of eucalyptus clones, located in the northeast region of the state of Bahia. The measurements were made at the ages $27,40,50,58,61,76,87,101,112,122,137,147,158$ and 165 months, with thinnings carried out at 58 and 142 months. Two diameter distribution models were used to prognosticate the diametric structures of the stands, starting from an observed distribution at ages 27 (before first thinning), 61 (after first thinning) and 147 (after second thinning) months. Three
\end{abstract}

Fonte de financiamento: Este estudo foi financiado pela Fundação de Amparo à Pesquisa de Minas Gerais (FAPEMIG), Coordenação de Aperfeiçoamento de Pessoal de Nível Superior - Brasil (CAPES) - Código Financeiro 001, e Universidade Federal dos Vales do Jequitinhonha e Mucuri (UFVJM).

Conflito de interesse: Nada a declarar.

Autor correspondente: barbosa_gabriella@hotmail.com

Recebido: 10 abril 2019.

Aceito: 17 outubro 2019.

Editor: Paulo Henrique Müller Silva.

(c) Este é um artigo publicado em acesso aberto (Open Access) sob a licença Creative Commons Attribution, que permite uso, distribuição e reprodução em qualquer meio, sem restrições desde que o trabalho original seja corretamente citado. 
systems were obtained according to the adjusting method of the Weibull function: System 1, using the linear approximation; Systems 2 and 3, using the maximum likelihood. The goodness of fit of prognosticated structures in relation to those observed was verified by the Kolmogorov-Smirnov test at $5 \%$ of significance. System 2 was the most suitable for practical applications for thinning in eucalypts. The maximum likelihood method should preferably be used when compared to linear approximation, ensuring a better quality in the prognoses of the diameter distribution.

Keywords: Weibull function; Diameter distribution; Maximum likelihood method; Linear approximation method.

\section{INTRODUÇÃO}

A crescente demanda por plantios de grande porte para o setor moveleiro, tem levado povoamentos de Eucalyptus a serem manejados sob regimes de desbastes. A avaliação de povoamentos desbastados, em geral, era realizada por modelos de crescimento e produção em nível de povoamento (Buckman, 1962). Com a evolução das técnicas de suporte à tomada de decisões para florestas plantadas e o surgimento de ferramentas mais específicas e eficazes, os modelos de distribuição de diâmetros (MDD) têm-se apresentado mais apropriados para avaliações em povoamentos desbastados.

Os MDD possuem maior flexibilidade e permitem realizar prognose e avaliação econômica de multiprodutos da madeira (Burkhart et al., 1981). Além disso, são capazes de estimar o número de árvores por hectare por classe de diâmetro em idades presente e futura. Os MDD utilizam uma função densidade de probabilidade (f.d.p.), sendo que a função Weibull tem sido a mais comumente empregada nessa modelagem, devido a sua grande flexibilidade e capacidade de descrever diversos tipos de distribuição (Bailey \& Dell, 1973; Campos \& Leite, 2017). Dentre as ferramentas mais conhecidas de ajuste de f.d.p. encontram-se a máxima verossimilhança e a aproximação linear.

De acordo com o objetivo do manejo são desenvolvidos MDD (Scolforo \& Machado, 1996; Eisfeld et al., 2005; Binoti et al., 2010, 2011; Stepka et al., 2011; Azevedo et al., 2016), que podem ser considerados boas ferramentas para a quantificação de diversos produtos que serão produzidos no povoamento. Além de possibilitarem a simulação de desbastes e da desrama artificial, podem retratar a veracidade de todo o sistema estudado.

A prognose da estrutura diamétrica da floresta pode ser realizada utilizando MDD, empregando-se dados confiáveis que contenham variáveis tradicionais de inventário, passíveis de serem correlacionadas. Além disso, é imprescindível a escolha da f.d.p. bem como o método de ajuste para a mesma, de modo a repercutir na precisão requerida. Assim, o objetivo deste estudo foi comparar dois modelos de distribuição de diâmetros para povoamentos de Eucalyptus grandis x urophylla submetidos a desbastes; e avaliar a influência de dois métodos de ajuste de funções densidade de probabilidade na qualidade das projeções de estruturas diamétricas.

\section{MATERIAL E MÉTODOS}

\section{Descrição dos dados}

Foram utilizados dados de um experimento cuja instalação foi realizada em povoamentos desbastados do híbrido de Eucalyptus grandis Hill ex Maiden x urophylla S.T. Blake, localizados na região Nordeste do estado da Bahia, pertencentes à empresa Bahia Specialty Cellulose. O experimento abrangeu locais com capacidades produtivas extremas e intermediárias, sendo localizado em três instalações, e composto por quatro tratamentos distribuídos em seis blocos, contendo dois blocos por instalação.

Foram totalizadas 48 unidades amostrais (contendo duas repetições por bloco) com área útil por parcela de aproximadamente $2.600 \mathrm{~m}^{2}$, sendo que o espaçamento inicial entre plantas foi de $3 \times 3 \mathrm{~m}$. Os tratamentos aplicados corresponderam a diferentes percentuais de remoção em área basal por desbaste: T1: redução de $20 \%$ da área basal presente, eliminando os piores indivíduos; T2: redução de $35 \%$ da área basal presente, eliminando os piores 
indivíduos; T3: redução de 50\% da área basal presente, eliminando os piores indivíduos; e T4: redução de $35 \%$ da área basal presente, eliminando os piores indivíduo, e desrama artificial nas árvores remanescentes, empregando-se $6,0 \mathrm{~m}$ de altura para esta atividade. O experimento descrito segue os princípios estabelecidos em Marshall \& Curtis (2002).

As parcelas permanentes foram mensuradas nas idades de 27, 40, 50, 58, 61, 76, 87, 101, 112, 122, 137, 147, 158 e 165 meses, sendo dois desbastes executados, aos 58 (primeiro desbaste) e 142 (segundo desbaste) meses. Em cada medição, para cada parcela foram registradas as seguintes características: razão de copa de cada árvore, que indica a proporção da altura total do indivíduo ocupada pela copa; altura total das quinze primeiras árvores; altura de cinco árvores dominantes e diâmetro a 1,30 $\mathrm{m}$ de altura (dap) de todas as árvores pertencentes às parcelas.

\section{Distribuição diamétrica estimada}

As frequências diamétricas foram estimadas por parcela em todas as idades consideradas, exceto nas idades 58 e 142 meses em que ocorrem desbastes, utilizando a função densidade de probabilidade Weibull de dois parâmetros. O parâmetro de locação (a) foi excluído da função. A amplitude de classe adotada para todos os ajustes foi de $2 \mathrm{~cm}$, a qual gerou bons resultados no trabalho de Araújo Júnior et al. (2010).

\section{Modelos de projeção dosparâmetros da função Weibull}

Foram ajustados doze modelos que projetam os parâmetros da função Weibull, sendo seis para o parâmetro de escala $\beta$ (Modelos 1, 2, 3, 4, 5 e 6) e seis para o parâmetro de forma $y$ (Modelos 7, 8, 9, 10, 11 e 12).

Modelos que projetam o parâmetro de escala;

Modelo 1: $\beta_{2}=\beta_{I}\left(\frac{I_{I}}{I_{2}}\right)+\theta_{I}\left(1-\frac{I_{I}}{I_{2}}\right) \operatorname{dmax}_{2}+\varepsilon$ (Nogueira, 2003)

Modelo 2: $\operatorname{Ln} \beta_{2}=\operatorname{Ln} \beta_{1}\left(\frac{I_{I}}{I_{2}}\right)+\theta_{1}\left(1-\frac{I_{1}}{I_{2}}\right) d m a x_{2}+\varepsilon$ (Binoti et al., 2010)

Modelo 3: $\beta_{2}=e^{\left(\theta_{1}+\theta_{2} \operatorname{dmax}_{2} \frac{\theta_{3}}{I_{2}}\right)}+\varepsilon$ (Adaptado de Leite et al., 2013)

Modelo 4: $\beta_{2}=\theta_{1} q_{1}+\varepsilon$ (Adaptado de Arce, 2005)

Modelo 5: $\beta_{2}=\frac{\theta_{1} q_{1}}{\theta_{2}+q_{1}}+\varepsilon$ (Adaptado de Nord-Larsen \& Cao, 2006)

Modelo 6: $\beta_{2}=\theta_{1}+\theta_{2} \operatorname{dmax}_{1}+\theta_{3}\left(\operatorname{dmax}_{1}-q_{1}\right)+\varepsilon$ (Adaptado de Campos \& Leite, 2017)

Modelos que projetam o parâmetro de forma;

Modelo 7: $L n \gamma_{2}=L n \gamma_{1} e^{-\theta_{1}\left(I_{2}^{\theta_{2}}-I_{1}^{\theta_{2}}\right)} \cdot{ }_{\varepsilon}$ (Nogueira, 2003)

Modelo 8: $L n \gamma_{2}=\theta_{1} e^{-\theta_{2}\left(I_{2}^{\theta_{3}}-I_{1}^{\theta_{3}}\right)}+\theta_{4} \beta_{2}+\theta_{5} L n \gamma_{1}+\varepsilon$ (Binoti et al., 2010)

Modelo 9: $\gamma_{2}=e^{\left(\theta_{1}+\theta_{2} L n \beta_{2}+\theta_{3} d m i n_{2}\right)}+\varepsilon$ (Adaptado de Leite et al., 2013)

Modelo 10: $\gamma_{2}=\theta_{1} q_{1}+\theta_{2} q_{1}{ }^{2}+\varepsilon$ (Adaptado de Arce, 2005)

Modelo 11: $\gamma_{2}=\frac{\theta_{1}}{1+\theta_{2} e^{\left(-\theta_{3} q_{l}\right)}}+\varepsilon$ (Adaptado de Nord-Larsen \& Cao, 2006)

Modelo 12: $\gamma_{2}=\theta_{1}+\theta_{2} d \max _{1}+\theta_{3}\left(\operatorname{dmax}_{1}-q_{1}\right)+\varepsilon$ (Adaptado de Campos \& Leite, 2017)

em que: $I_{2}=$ idade futura, em meses; $I_{I}=$ idade atual, em meses; $\gamma_{2}=$ parâmetro de forma da função Weibull em uma idade futura; $\gamma_{l}=$ parâmetro de forma da função Weibull em uma idade atual; $\beta_{2}=$ parâmetro de escala da função Weibull em uma idade futura; $\beta_{1}=$ parâmetro de escala da função Weibull em uma idade atual; $d \max _{2}=$ diâmetro máximo em uma idade futura, em $\mathrm{cm} ; d \max _{1}=$ diâmetro máximo em uma idade atual, em $\mathrm{cm} ; d_{\min }$ = diâmetro mínimo em uma idade futura, em $\mathrm{cm} ; q_{l}=$ diâmetro médio em uma idade atual, em $\mathrm{cm} ; e=$ base neperiana; 
Ln = logaritmo neperiano; $\varepsilon=$ erros aleatórios independentes, com média zero e variância constante; e $\theta_{i}=$ parâmetros dos modelos.

Nos ajustes foram utilizados dados oriundos dos ajustes da f.d.p. Weibull por parcela utilizando os métodos da máxima verossimilhança e da aproximação linear. Estes ajustes foram realizados com o intuito de verificar a eficiência dos modelos testados, bem como a influência dos métodos da máxima verossimilhança e da aproximação linear nos valores projetados.

Para o ajuste dos modelos estatísticos utilizaram-se o Método dos Mínimos Quadrados Ordinários (para modelos lineares) e o Método dos Mínimos Quadrados Não-Lineares (para modelos não-lineares). Os ajustes foram efetuados no software Statistica 10 (StatSoft, Inc., 2010), empregando-se o procedimento iterativo Quasi-Newton. Para fins de avaliação, foram obtidos os coeficientes de correlação $\left(R_{\hat{Y Y}}\right)$ para todas as equações ajustadas, bem como os respectivos gráficos de resíduo.

\section{Modelos de distribuição diamétrica - MDD}

Foram utilizados dois MDD para projetar as estruturas diamétricas dos povoamentos desbastados de eucalipto. A distribuição diamétrica foi projetada partindo-se de uma idade atual para uma idade futura, utilizando-se sempre uma distribuição inicial observada. As projeções foram realizadas por parcela com a distribuição observada da primeira medição antes do desbaste (27 meses), após o primeiro desbaste $(61$ meses) e após o segundo desbaste (147 meses).

O primeiro MDD testado foi desenvolvido por Nogueira (2003) e pode ser utilizado para projetar estruturas diamétricas de povoamentos equiâneos de eucalipto não desbastados ou submetidos a desbaste. Os parâmetros de escala e forma ( $\beta$ e $y$ ) que são entradas no modelo, foram estimados por unidade amostral com o ajuste da função Weibull pelos métodos da aproximação linear (Sistema 1) e da máxima verossimilhança (Sistema 2). O MDD proposto é dado pelo seguinte sistema de equações:

$$
\begin{aligned}
& d \min _{2}=d \operatorname{din}_{1} e^{-\theta_{1}\left(I_{2}^{\theta_{2}}-I_{I}^{\theta_{2}}\right)} \cdot \varepsilon \\
& \operatorname{Ln\gamma _{2}}=\operatorname{Ln\gamma _{1}} e^{-\theta_{3}\left(I_{2}^{\theta_{4}}-I_{1}^{\theta_{4}}\right)} \cdot \varepsilon \\
& \operatorname{dmax}_{2}=\operatorname{dmax}_{1}\left(\frac{I_{1}}{I_{2}}\right)+\theta_{5}\left(1-\frac{I_{I}}{I_{2}}\right) \beta_{1}+\varepsilon \\
& \beta_{2}=\beta_{I}\left(\frac{I_{I}}{I_{2}}\right)+\theta_{6}\left(1-\frac{I_{I}}{I_{2}}\right) d m a x_{2}+\varepsilon \\
& N_{2}=N_{I} e^{-\theta_{7}\left(I_{2}^{\theta_{-}}-I_{1}^{\theta_{8}}\right)} \cdot \varepsilon \text { (Antes do desbaste) } \\
& N_{2}=N_{I} \text { (Após o desbaste) }
\end{aligned}
$$

em que:

$I_{2}=$ idade futura, em meses; $I_{1}=$ idade atual, em meses; $\gamma_{2}=$ parâmetro de forma da função Weibull na idade futura; $\gamma_{1}=$ parâmetro de forma da função Weibull na idade atual; $\beta_{2}=$ parâmetro de escala da função Weibull na idade futura; $\beta_{I}=$ parâmetro de escala da função Weibull na idade atual; $d \max x_{2}=$ diâmetro máximo na idade futura, em cm; $d \max x_{l}=$ diâmetro máximo na idade atual, 
em cm; $d \min _{2}$ = diâmetro mínimo na idade futura, em cm; $d \min _{1}$ = diâmetro mínimo na idade atual, em $\mathrm{cm} ; N_{2}=$ número de árvores por ha na idade futura; $N_{l}=$ número de árvores por ha na idade atual; $e=$ base neperiana; $L n=$ logaritmo neperiano; $\varepsilon=$ erros aleatórios independentes, com média zero e variância constante; e $\theta_{i}=$ parâmetros dos modelos.

Uma característica importante relacionada com a consistência do sistema apresentado é que quando a idade futura for igual à idade atual, a distribuição diamétrica projetada é igual à distribuição observada. Em outras palavras, os valores preditos dos parâmetros da função Weibull permanecem os mesmos quando essa situação ocorre $\left(I_{2}=l_{1}\right)$, assegurando a lógica e o realismo biológico. Vale ressaltar que o sistema não considera mortalidade após o desbaste, ou seja, há eliminação da mortalidade natural das árvores, que é um dos principais objetivos deste trato silvicultural.

O segundo MDD testado foi adaptado de Binoti et al. (2010) e é dado pelo seguinte sistema de equações:

$$
\begin{aligned}
& \operatorname{dmin}_{2}=\operatorname{dmin}_{1} e^{-\theta_{l}\left(I_{2}^{\theta_{2}}-I_{I}^{\theta_{2}}\right)} \cdot \varepsilon \\
& d \max _{2}=\operatorname{dmax}_{1}\left(\frac{I_{1}}{I_{2}}\right)+\theta_{3}\left(1-\frac{I_{1}}{I_{2}}\right) q_{I}+\varepsilon \\
& \operatorname{Ln} \beta_{2}=\operatorname{Ln} \beta_{1}\left(\frac{I_{1}}{I_{2}}\right)+\theta_{4}\left(1-\frac{I_{1}}{I_{2}}\right) d \max _{2}+\varepsilon \\
& L n \gamma_{2}=\theta_{5} e^{-\theta_{6}\left(I_{2}^{\theta_{7}}-I_{1}^{\theta_{7}}\right)}+\theta_{8} \beta_{2}+\theta_{9} L n \gamma_{1}+\varepsilon
\end{aligned}
$$

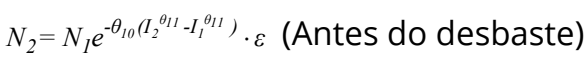

$$
\begin{aligned}
& N_{2}=N_{l} \text { (Após o desbaste) }
\end{aligned}
$$

em que:

$q_{l}=$ diâmetro médio em uma idade atual, em cm; $I_{2}=$ idade futura, em meses; $I_{l}=$ idade atual, em meses; $\gamma_{2}=$ parâmetro de forma da função Weibull na idade futura; $\gamma_{1}=$ parâmetro de forma da função Weibull na idade atual; $\beta_{2}=$ parâmetro de escala da função Weibull na idade futura; $\beta_{1}=$ parâmetro de escala da função Weibull na idade atual; $d \max _{2}=$ diâmetro máximo na idade futura, em cm; $d \max _{1}=$ diâmetro máximo na idade atual, em cm; $d \min _{2}=$ diâmetro mínimo na idade futura, em $\mathrm{cm} ; d \min _{1}=$ diâmetro mínimo na idade atual, em $\mathrm{cm} ; N_{2}=$ número de árvores por ha na idade futura; $N_{l}=$ número de árvores por ha na idade atual; $e=$ base neperiana; $L n=$ logaritmo neperiano; $\varepsilon=$ erros aleatórios independentes, com média zero e variância constante; e $\theta_{i}=$ parâmetros dos modelos.

Os parâmetros de escala e forma ( $\beta$ e $y$ ) que, são entradas no modelo, foram estimados por parcela com o ajuste da função Weibull pelo método da máxima verossimilhança (Sistema 3).

O método da aproximação linear empregado foi o descrito por Campos \& Leite (2017). E o método da máxima verossimilhança utilizado foi definido conforme Gove \& Fairweather (1989).

Para o ajuste dos modelos que compõem os sistemas propostos, foram também utilizados o Método dos Mínimos Quadrados Ordinários (para modelos lineares) e o Método dos Mínimos Quadrados Não-Lineares (para modelos não-lineares). Os ajustes foram 
efetuados no software Statistica 10 (StatSoft, Inc., 2010), empregando-se o processo iterativo Quasi-Newton, e a avaliação realizada pelos coeficientes de correlação $\left(R_{\hat{Y} Y}\right)$ para todas as equações ajustadas.

Para verificar a aderência das estruturas prognosticadas pelos modelos de distribuição diamétrica em relação aos dados observados, utilizou-se o teste não paramétrico de Kolmogorov-Smirnov a 5\% de significância. Este compara a frequência acumulada observada com a frequência acumulada projetada, em que a estatística do teste é obtida na classe que corresponde ao maior valor divergente.

$D=\operatorname{má} x|F o(x)-F e(x)|$

em que:

$D$ = estatística do teste, ou seja, diferença máxima absoluta; $F o(x)=$ frequência acumulada observada; e $F e(x)$ = frequência acumulada projetada.

\section{RESULTADOS E DISCUSSÃO}

As tendências para os parâmetros de escala e de forma da função Weibull estimados variaram de acordo com a variável de povoamento utilizada, e foram influenciadas pelo método de ajuste de função densidade de probabilidade empregado (Figuras 1 e 2).

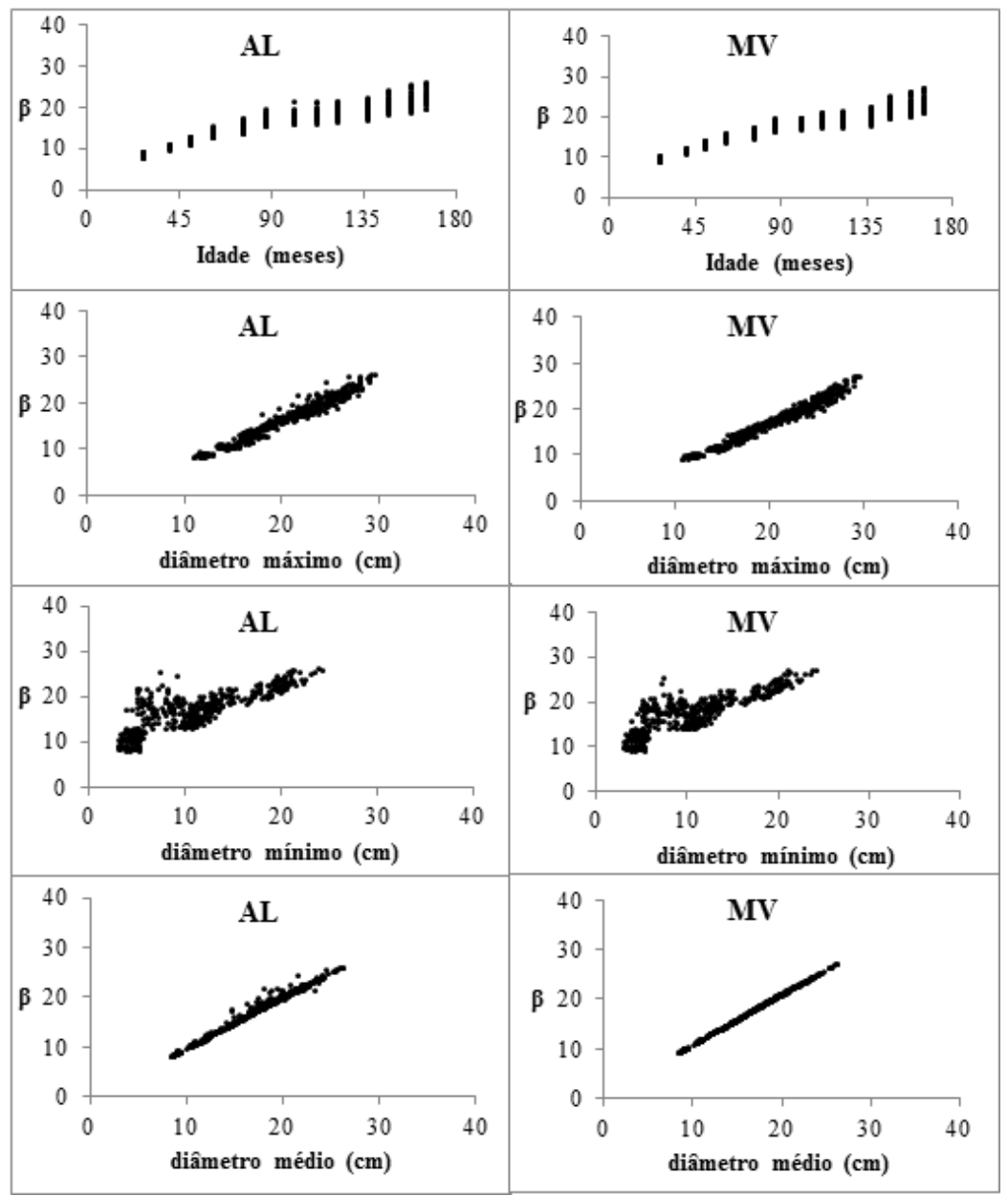

Figura 1. Tendências para os valores de parâmetro de escala $(\beta)$ em função das variáveis de povoamento idade, diâmetro máximo, diâmetro mínimo e diâmetro médio, obtidos pelos ajustes da função Weibull por parcela e idade de medição nos métodos da aproximação linear $(\mathrm{AL})$ e máxima verossimilhança (MV). 


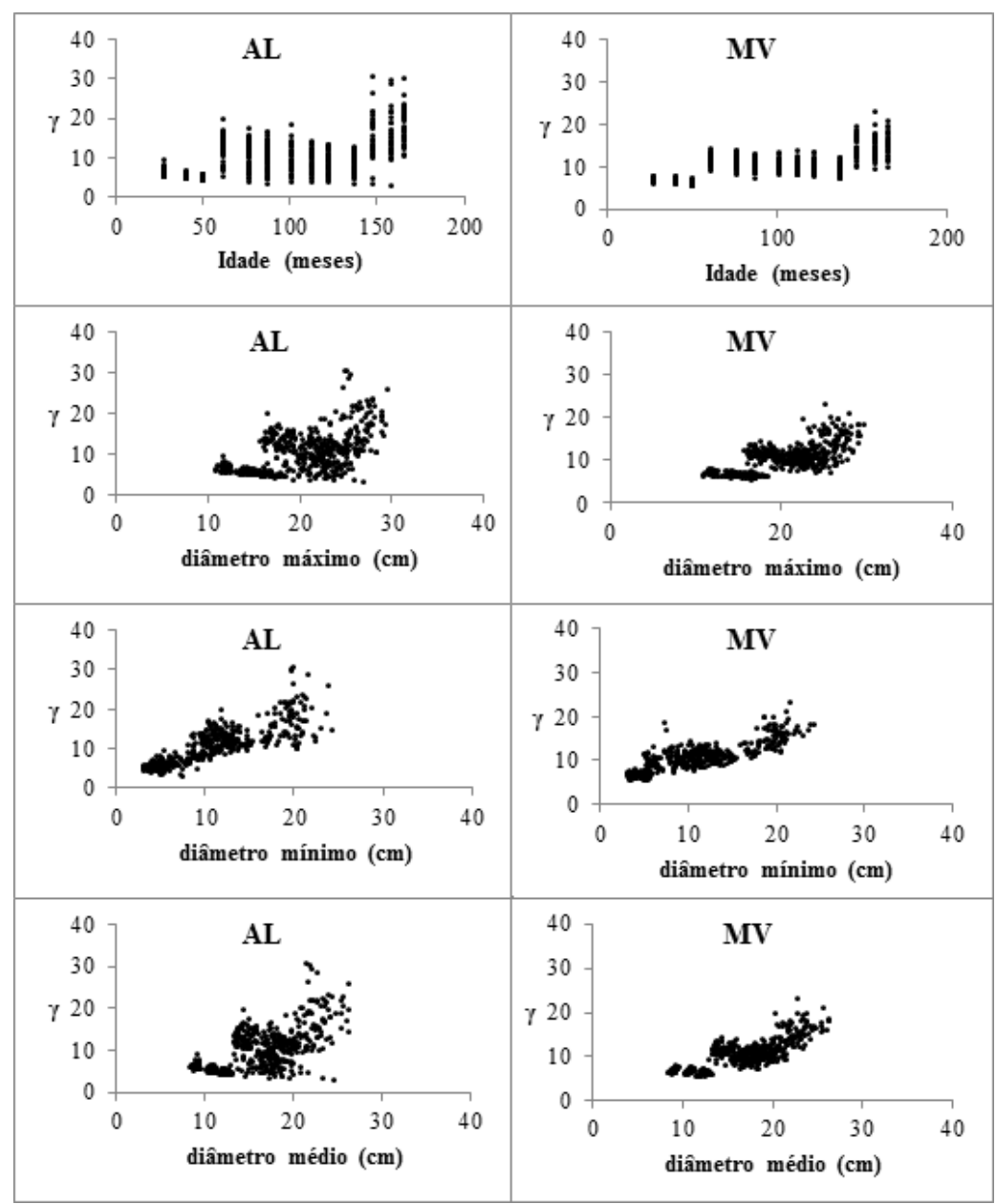

Figura 2. Tendências para os valores de parâmetro de forma (y) em função em função das variáveis de povoamento idade, diâmetro máximo, diâmetro mínimo e diâmetro médio, obtidos pelos ajustes da função Weibull por parcela e idade de medição nos métodos da aproximação linear (AL) e máxima verossimilhança $(\mathrm{MV})$.

Ao analisar os gráficos, observou-se que há indícios para se afirmar que o método da aproximação linear gera comportamentos menos definidos em detrimento ao uso da máxima verossimilhança. Ou seja, os pontos que definem as tendências encontram-se mais próximos um do outro no segundo método. O parâmetro de escala apresentou menor relação com a variável diâmetro mínimo e maior relação com o diâmetro médio quadrático, ao passo que a idade e o diâmetro máximo foram os intermediários neste quesito (Figura 1).

Quanto ao parâmetro de forma, este apresentou maior relação com o diâmetro mínimo (Figura 2). É possível verificar que esse parâmetro é dependente do efeito do desbaste, ou seja, o mesmo aumenta nas idades após ocorrido este tratamento silvicultural (61 e 147 meses).

Considerando os métodos da aproximação linear e máxima verossimilhança quando se analisa um mesmo modelo e compara as correlações entre os dois métodos de ajuste, no geral, verificam-se que os valores das estatísticas pelo método da aproximação linear são inferiores àqueles gerados pelo outro método. Este fato é um indicativo de superioridade da máxima verossimilhança.

Analisando-se a distribuição dos resíduos dos modelos que estimam o parâmetro de escala (modelos 1-6) e considerando um mesmo método de ajuste da Weibull, é possível afirmar que não existem diferenças que apontem para a escolha de um ou outro a ser utilizado (Figura 3). O mesmo ocorre para o parâmetro de forma (modelos 7-12). Portanto, qualquer um dos modelos testados possui potencial para compor um sistema que realiza a prognose da distribuição diamétrica. 

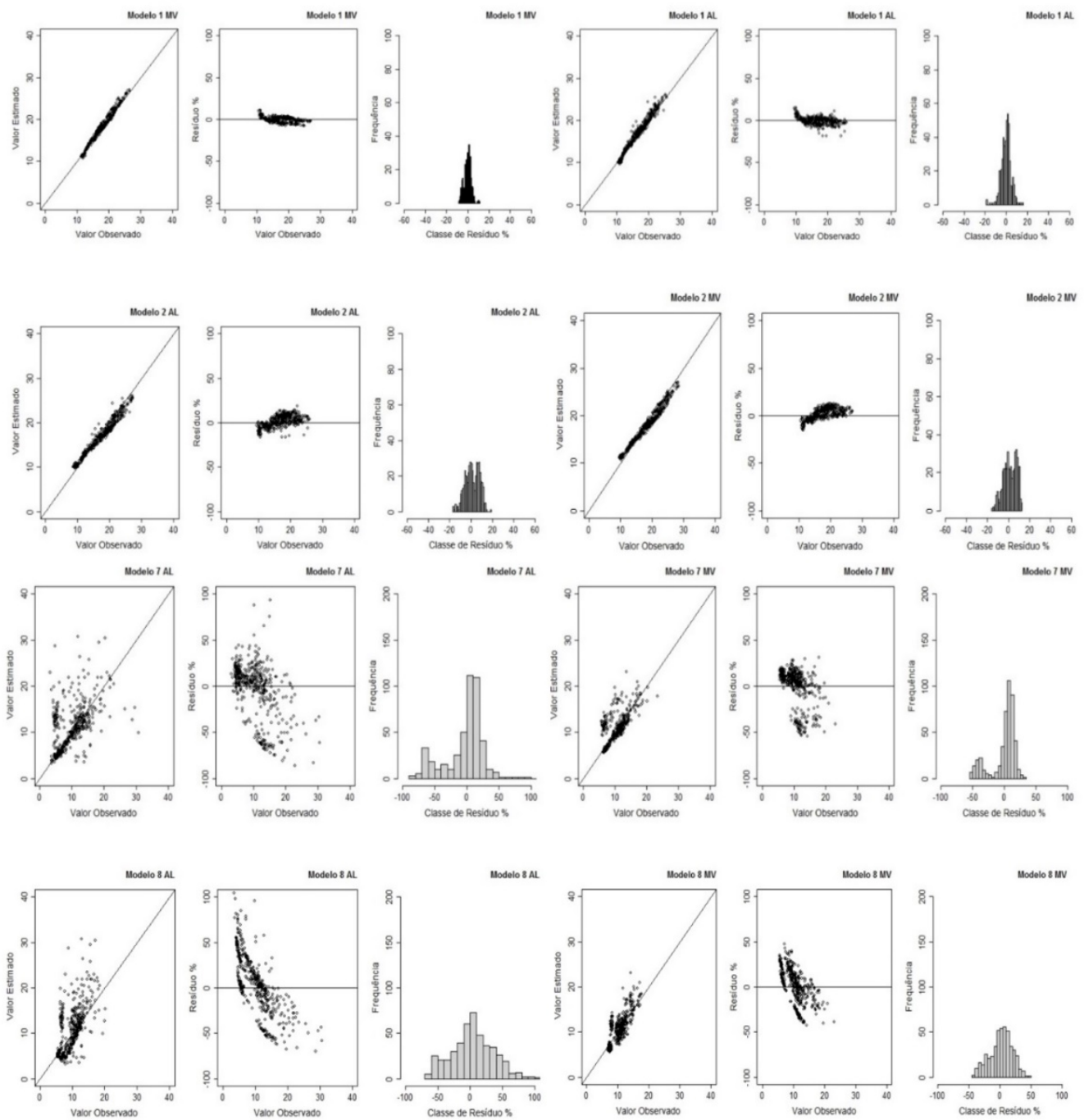

Figura 3. Distribuição de resíduos para alguns modelos de projeção dos parâmetros de escala (modelos 1, 2) e forma (modelos 7, 8), estimados com dados oriundos do ajuste da Weibull nos métodos da aproximação linear (AL) e máxima verossimilhança (MV).

Em todos os casos, partindo-se de um mesmo modelo e comparando entre os métodos de ajuste da função Weibull, a aproximação linear foi inferior em detrimento à máxima verossimilhança. Isto comprova a superioridade deste método e uma das razões para recomendá-lo.

Os resultados dos ajustes dos dois modelos de distribuição de diâmetros, bem como as estatísticas consideradas, estão apresentados na Tabela 1.

Os maiores valores dos coeficientes de correlação estão presentes nos Sistemas 2 e 3, os quais tiveram equações baseadas nos parâmetros de escala e forma obtidos pelo método da máxima verossimilhança. Embora no Sistema 1 tenha sido empregado o método da aproximação linear para estimação dos mesmos parâmetros, os resultados dos coeficientes dos modelos matemáticos desse sistema foram muito próximos dos estimados no Sistema 2.

Foram efetuadas 512 projeções para cada sistema relatado, totalizando 1536 estimativas de frequências por classe de diâmetro. O melhor desempenho ocorreu no Sistema 2, no qual a porcentagem de projeções não significativas pelo teste de Komolgorov-Smirnov atingiu $99,80 \%$. O pior desempenho foi encontrado no Sistema 3, no qual o número de significativos foi relativamente expressivo $(7,03 \%)$. Dentre todas as projeções executadas nesse último sistema, 36 delas não aderiram aos valores de frequência observada. O Sistema 1 apresentou $98,44 \%$ de projeções não significativas pelo teste de Komolgorov-Smirnov. 
Tabela 1. Coeficientes das equações ajustadas dos três sistemas propostos e os respectivos coeficientes de correlação $\left(R_{\hat{Y} Y}\right)$

\begin{tabular}{|c|c|}
\hline \multicolumn{2}{|l|}{ Sistema 1} \\
\hline$d \min _{2}=\operatorname{dmin}_{1} e^{-(-0,004152)\left(I_{2}^{1,000492}-I_{I}^{1,090492}\right)}$ & $R_{\hat{Y} Y}=0,8371$ \\
\hline $\operatorname{Ln} \gamma_{2}=\operatorname{Ln} \gamma_{1} e^{-(111,941850)\left(I_{2}^{-0,000189}-I_{I}^{-0,000189}\right)}$ & $R_{\hat{Y} Y}=0,3879$ \\
\hline$d \max _{2}=\operatorname{dmax}_{1}\left(\frac{I_{1}}{I_{2}}\right)+2,004974\left(1-\frac{I_{1}}{I_{2}}\right) \beta_{I}$ & $R_{\hat{Y} Y}=0,9791$ \\
\hline$\beta_{2}=\beta_{1}\left(\frac{I_{1}}{I_{2}}\right)+1,136966\left(1-\frac{I_{1}}{I_{2}}\right) d \max _{2}$ & $R_{\hat{Y Y}}=0,9818$ \\
\hline$N_{2}=N_{1} e^{-(273,789776)\left(I_{2}^{0.0000031}-I_{l}^{0.000031}\right)}$ (Antes do desbaste) & $R_{\hat{Y Y}}=0,9960$ \\
\hline \multicolumn{2}{|l|}{ Sistema 2} \\
\hline$d \min _{2}=d \min _{1} e^{-(-0,004152)\left(I_{2}^{1.090492}-I_{1}^{1,0094992}\right)}$ & $R_{\hat{Y} Y}=0,8371$ \\
\hline $\operatorname{Ln} \gamma_{2}=\operatorname{Ln} \gamma_{1} e^{-(111,955491)\left(I_{2}^{-0,0000808}-I_{I}^{-0,000808}\right)}$ & $R_{\hat{Y Y}}=0,5846$ \\
\hline$d m a x_{2}=\operatorname{dmax}_{1}\left(\frac{I_{1}}{I_{2}}\right)+1,873855\left(1-\frac{I_{1}}{I_{2}}\right) \beta_{1}$ & $R_{\hat{Y} Y}=0,9815$ \\
\hline$\beta_{2}=\beta_{1}\left(\frac{I_{1}}{I_{2}}\right)+1,180514\left(1-\frac{I_{1}}{I_{2}}\right) d \max _{2}$ & $R_{\hat{Y} Y}=0,9888$ \\
\hline$N_{2}=N_{l} e^{-(273,789776)\left(I_{2}^{0,000031}-I_{l}^{0,000031}\right)}$ (Antes do desbaste) & $R_{\hat{Y Y}}=0,9960$ \\
\hline \multicolumn{2}{|l|}{ Sistema 3} \\
\hline$d \min _{2}=\operatorname{dmin}_{1} e^{-(-0,004152)\left(I_{2}^{1,000492}-I_{1}^{1,090492}\right)}$ & $R_{\hat{Y Y}}=0,8371$ \\
\hline $\operatorname{dmax}_{2}=\operatorname{dmax}_{I}\left(\frac{I_{1}}{I_{2}}\right)+1,969967\left(1-\frac{I_{I}}{I_{2}}\right) q_{1}$ & $R_{\hat{Y Y}}=0,9811$ \\
\hline $\operatorname{Ln} \beta_{2}=\operatorname{Ln} \beta_{1}\left(\frac{I_{1}}{I_{2}}\right)+0,172923\left(1-\frac{I_{1}}{I_{2}}\right) d \max x_{2}$ & $R_{\hat{Y Y}}=0,9609$ \\
\hline $\operatorname{Ln} \gamma_{2}=1,062149 e^{-(1189,309848)\left(I_{2}^{0,000108}-I_{1} I_{1}^{0,000108}\right)}+0,040613 \beta_{2}+0,251033 \operatorname{Ln} \gamma_{1}$ & $R_{\hat{Y} Y}=0,7782$ \\
\hline$N_{2}=N_{1} e^{-(273,789776)\left(I_{2}^{0,0000031}-I_{1} I_{1}^{0,000031}\right)}$ (Antes do desbaste) & $R_{\hat{Y} Y}=0,9960$ \\
\hline
\end{tabular}

em que: Sistemas 1 e 2 = modelo de distribuição diamétrica desenvolvido por Nogueira (2003); Sistema 3 = modelo de distribuição diamétrica adaptado de Binoti et al. (2010).

Para demonstrar as tendências da prognose em relação às estruturas diamétricas observadas, foram traçadas as curvas para algumas parcelas selecionadas de forma que representasse os três sistemas testados (Figuras 4 e 5).

Como o Sistema 2 foi superior em termos de projeções não significativas, este foi confrontado com o Sistema 1 que foi imediatamente inferior. O mesmo foi executado para os Sistemas 1 e 3, seguindo a mesma lógica mencionada anteriormente.

Ao analisar algumas curvas geradas pelos Sistemas 1 e 2 (Figura 4), observou-se que o Sistema 2, no qual foi utilizado o método da máxima verossimilhança, delineou tendências acompanhando melhor os valores observados. Por outro lado, o Sistema 1 tendeu a subestimar os resultados. Embora no Sistema 3 também tenha sido empregado o método da máxima verossimilhança, as prognoses tenderam a superestimar os resultados de distribuição diamétrica (Figura 5).

$\mathrm{Na}$ escolha de um modelo de distribuição de diâmetros para aplicações práticas, é fundamental que o conjunto de equações que compõem o sistema seja consistente. Assim, a forma que são correlacionados os parâmetros da função Weibull com os atributos do povoamento é crucial para um bom desempenho de todo o modelo. Isto pode ser verificado quando se trata do Sistema 3 que, apesar de ter sido empregado o método da máxima verossimilhança para obtenção dos parâmetros de escala e forma, foi o menos eficiente. 
Nogueira (2003), após testar diversas conformações de modelos de distribuição diamétrica, comprovou também que o conjunto de equações selecionado influencia diretamente na qualidade das projeções.

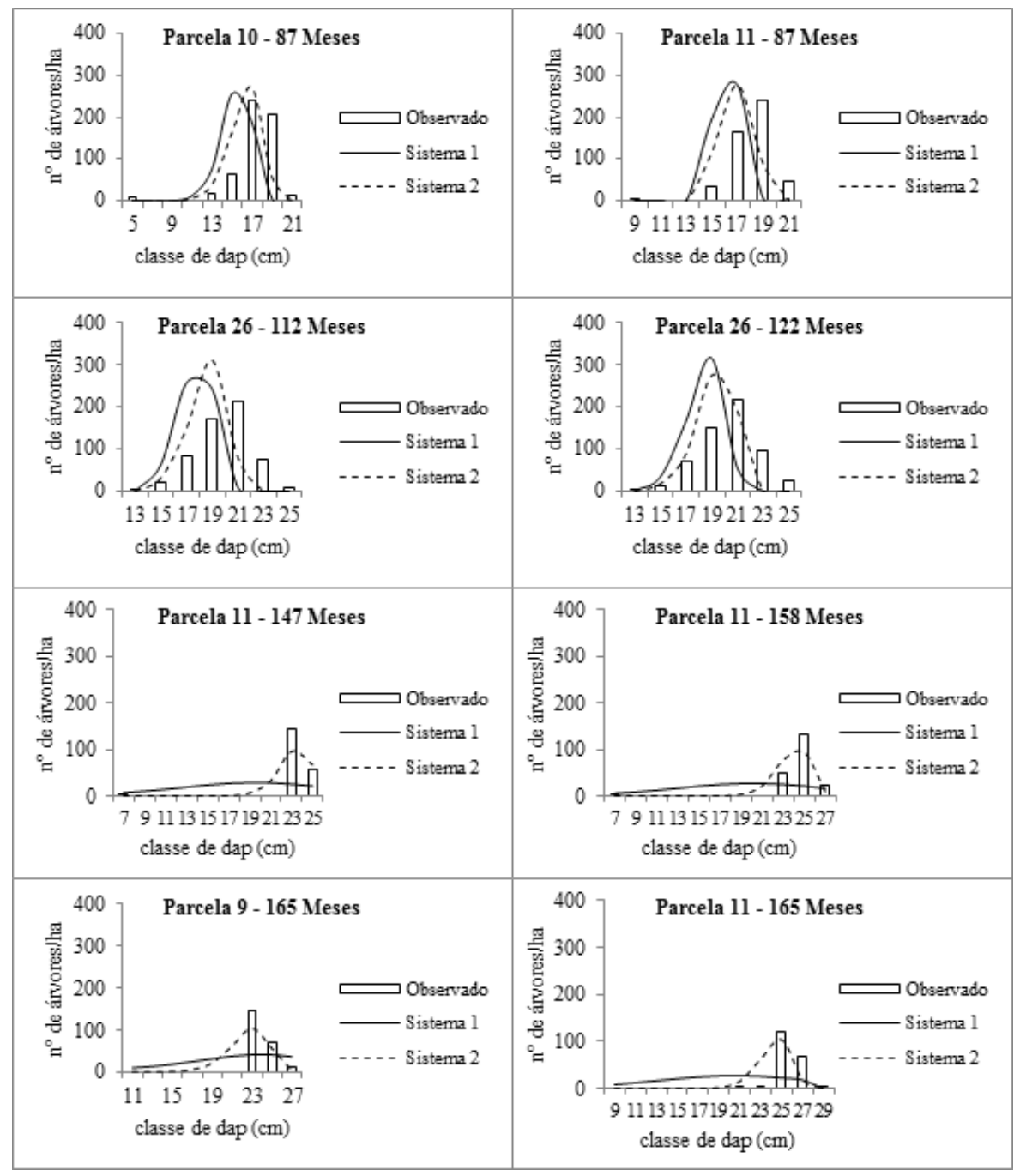

Figura 4. Distribuições diamétricas observadas e projetadas para povoamentos de Eucalyptus grandis $x$ urophylla submetidos a desbastes pelos Sistemas 1 e 2 em algumas parcelas, sendo aquelas realizadas no Sistema 1 significativas pelo teste de Kolmogorov-Smirnov a 5\% de significância.

O Sistema 2 foi o mais adequado para fins de manejo de povoamentos de eucalipto submetidos a desbastes no longo prazo. Conforme foi visto, esse sistema foi o único que proporcionou estimativas de prognose mais confiáveis em idades mais avançadas da floresta. Portanto, dentre os três sistemas analisados, recomenda-se apenas a aplicação do Sistema 2 para fins de planejamento tático de uma empresa florestal que possua produtos destinados à serraria (Bettinger et al., 2009).

O método da aproximação linear foi menos eficiente em detrimento ao emprego da máxima verossimilhança em termos de projeção da distribuição diamétrica. Comprova-se esta afirmação pelas prognoses executadas nos Sistemas 1 e 2 que, partindo-se de um mesmo conjunto de equações, é evidente a diferença na qualidade das projeções. Esse resultado corrobora com os de outros trabalhos (Araújo Júnior et al., 2010; Castro et al., 2017), os quais reportaram resultados satisfatórios para o método da máxima verossimilhança no ajuste da função densidade de probabilidade Weibull para povoamentos de eucalipto. Ainda, nas equações testadas que projetam os parâmetros de escala e de forma, houve superioridade 
daquelas que se basearam em dados oriundos de ajustes por meio da máxima verossimilhança.

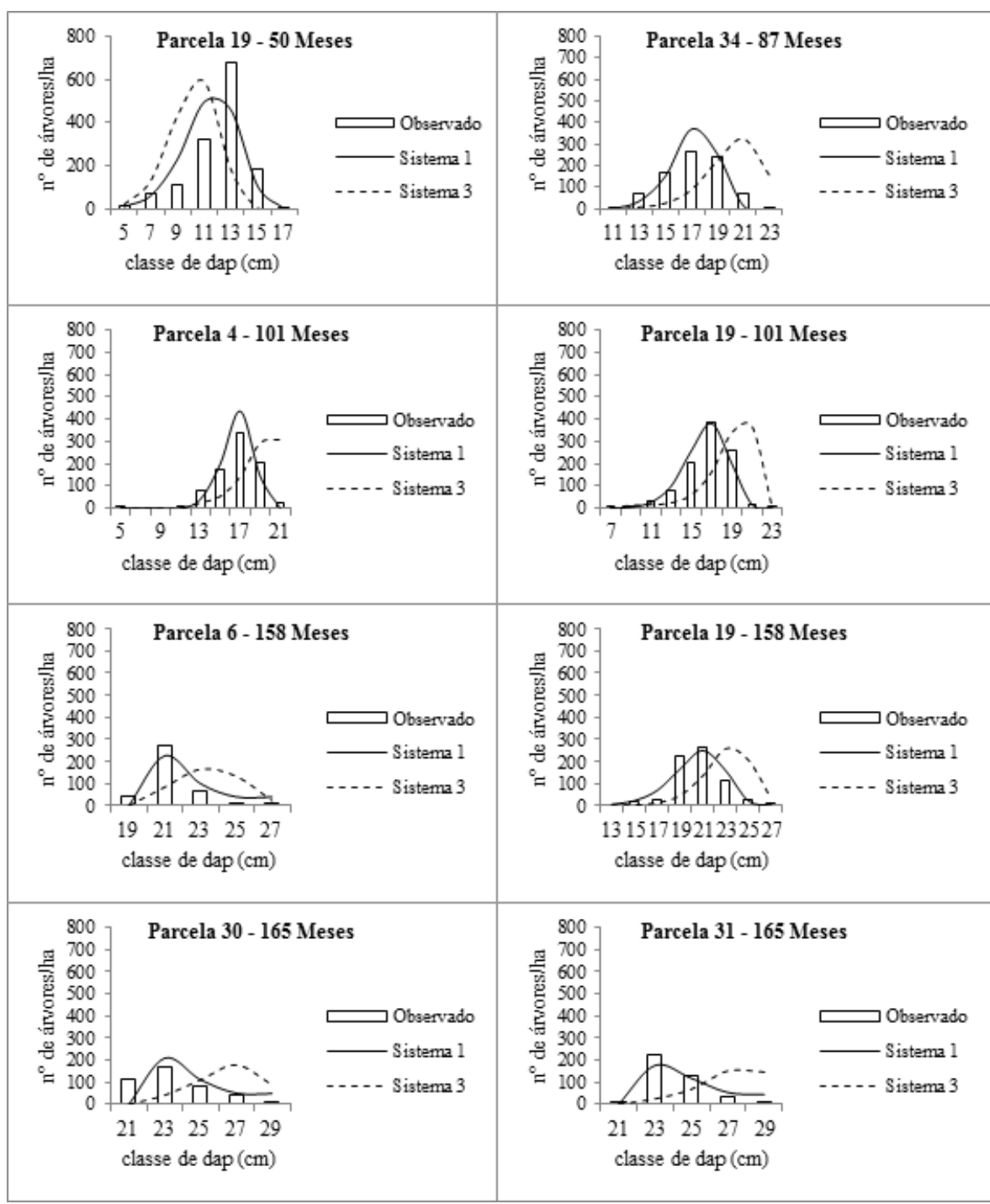

Figura 5. Distribuições diamétricas observadas e projetadas para povoamentos de Eucalyptus grandis $\mathrm{x}$ urophylla submetidos a desbastes pelos Sistemas 1 e 3 para algumas parcelas, sendo aquelas realizadas pelo Sistema 3 significativas pelo teste de Kolmogorov-Smirnov a 5\% de significância.

Portanto, se um determinado método ajusta melhor a função densidade de probabilidade escolhida, este método também ocasiona projeções mais adequadas por um modelo de distribuição diamétrica.

\section{CONCLUSÃO}

Ao utilizar uma determinada função densidade de probabilidade, mesmo empregandose o melhor método de ajuste, as equações que compõem o modelo de distribuição de diâmetros são as que podem limitar a precisão das estimativas.

Dentre os modelos de distribuição diamétrica comparados, o Sistema 2 se mostrou o mais promissor para aplicações práticas nas quais envolvem desbastes seletivos em povoamentos de eucalipto.

O método da máxima verossimilhança deve ser preferencialmente utilizado quando comparado à aproximação linear, garantindo melhor qualidade nas prognoses da distribuição diamétrica. 


\section{REFERÊNCIAS}

Araújo Júnior, A. C., Nogueira, G. S., Oliveira, M. L. R., Miranda, R. O. V., Castro, R. V. O., \& Pelli, E. (2010). Projeção da distribuição diamétrica de povoamentos de eucalipto em diferentes amplitudes de classe. Pesquisa Agropecuária Brasileira, 45(11), 1275-1281. http://dx.doi.org/10.1590/S0100-204X2010001100007.

Arce, J. E. (2005). Modelagem da estrutura de florestas clonais de Populus deltoides Marsh. através de distribuições diamétricas probabilísticas. Ciência Florestal, 14(1), 149-164. http://dx.doi.org/10.5902/198050981790.

Azevedo, G. B., Oliveira, E. K. B., Azevedo, G. T. O. S., Buchmann, H. M., Miguel, E. P., \& Rezende, A. V. (2016). Modelagem da produção em nível de povoamento e por distribuição diamétrica em plantios de eucalipto. Scientia Forestalis, Piracicaba, 44(110), 383-392.

Bailey, R. L., \& Dell, T. R. (1973). Quantifying diameter distributions with the Weibull function. Forest Science, 19(2), 97104.

Bettinger, P., Boston, K., Siry, J. P., \& Grebner, D. L. (2009). Forest management and planning (360 p.). Burlington: Academic Press.

Binoti, D. H. B., Binoti, M. L. M. S., Leite, H. G., Garcia, S. L. R., \& Cruz, J. P. (2011). Modelos de distribuição diamétrica para povoamentos desbastados de eucalipto utilizando a função hiperbólica. Revista Árvore, 35(5), 1069-1076. http://dx.doi.org/10.1590/S0100-67622011000600013.

Binoti, D. H. B., Leite, H. G., Nogueira, G. S., Silva, M. L. M., Garcia, S. L. R., \& Cruz, J. P. (2010). Uso da função Weibull de três parâmetros em um modelo de distribuição diamétrica para plantios de eucalipto submetidos a desbaste. Revista Árvore, 34(1), 147-156. http://dx.doi.org/10.1590/S0100-67622010000100016.

Buckman, R. E. (1962). Growth and yield of red pine in Minnesota (Technical Bulletin, No. 1272). Washington: USDA.

Burkhart, H. E., Cao, Q. V., \& Ware, K. D. (1981). A comparison of growth and yield prediction models for loblolly pine (59 p.). Virginia: Virginia Virginia Polytechnic Institute and State University.

Campos, J. C. C., \& Leite, H. G. (2017). Mensuração florestal: perguntas e respostas (5. ed.). Viçosa: Editora UFV.

Castro, R. V. O., Bruzinga, J. S., Mota, F. C. M., Azevedo, T. O. S., Azevedo, G. B., Leite, H. G., \& Silva, A. A. L. (2017). Procedimentos de ajuste da função Weibull para prognose da distribuição diamétrica de povoamentos de eucalipto. Scientia Agraria, 16(4), 528-534.

Eisfeld, R. D. L., Sanquetta, C. R., Arce, J. E., Maestri, R., \& Weber, K. S. (2005). Modelagem do crescimento e da produção de Pinus taeda L. por meio de função probabilística. Floresta, 35(2), 317-328. http://dx.doi.org/10.5380/rf.v35i2.4619.

Gove, J. H., \& Fairweather, S. E. (1989). Maximum-likelihood estimation of Weibull function parameters using a general interactive optimizer and grouped data. Forest Ecology and Management, 28(1), 61-69. http://dx.doi.org/10.1016/0378-1127(89)90074-1.

Leite, H. G., Alcântara, A. E. M., Binot, D. H. B., Oliveira Neto, R. R., \& Silva, M. L. M. (2013). Comparação entre predição e projeção da distribuição de diâmetros de povoamentos de eucalipto submetidos a desbastes. Revista Árvore, 37(2), 321-328. http://dx.doi.org/10.1590/S0100-67622013000200013.

Marshall, D. D., \& Curtis, R. O. (2002). Levels-of-growing-stock cooperative study in Douglas-fir. Portland: USDA, Pacific Northwest Research Station, Forest Service.

Nogueira, G. S. (2003). Modelagem do crescimento e da produção de povoamentos de Eucalyptus sp. e de Tectona grandis submetidos a desbaste (Tese de doutorado). Universidade Federal de Viçosa, Viçosa.

Nord-Larsen, T., \& Cao, Q. V. (2006). A diameter distribution model for even-aged beech in Denmark. Forest Ecology and Management, 231(1-3), 218-225. http://dx.doi.org/10.1016/j.foreco.2006.05.054.

Scolforo, J. R. S., \& Machado, S. A. (1996). Um sistema de crescimento e produção com simulador de desbaste. Scientia Forestalis, 50, 51-64.

StatSoft, Inc. (2010). Statistica (Data Analysis Software System), version 10.0. Tulsa. Recuperado em 10 de abril de 2019, de http://www.statsoft.com

Stepka, T. F., Lisboa, G. S., \& Kurchaidt, S. S. (2011). Funções densidade de probabilidade para a estimativa da distribuição diamétrica em povoamento de Eucalyptus sp na região centro sul do Paraná. Ambiência, 7(3), 429439. http://dx.doi.org/10.5777/ambiencia.2011.03.02.

Contribuição dos autores: EGS: conceituação, curadoria de Dados, análise formal, investigação, metodologia, administração do projeto, visualização, escrita - Primeira redação, GSN: conceituação, análise formal, obtenção de financiamento, administração do projeto, recursos, supervisão, escrita - primeira redação, HGL: conceituação, software, validação, MLRO: conceituação, análise formal, obtenção de financiamento, recursos, software, supervisão, GPB: análise formal, investigação, validação, visualização, escrita - primeira redação, escrita - revisão e edição, ETL: validação, visualização, escrita - primeira redação, escrita - revisão e edição. 